\title{
Dual and broadband power dividers at microwave frequencies based on composite right/left handed (CRLH) lattice networks
}

\author{
P. Vélez*, J.Bonache, F. Martín \\ GEMMA/CIMITEC, Departament d'Enginyeria Electrònica, Universitat \\ Autònoma de Barcelona, 08193 Bellaterra (Barcelona), Spain.
}

*Corresponding author (email): paris.velez@uab.cat Phone Number: +34935813513

\begin{abstract}
This paper proposes a dual-band power divider operating at $\mathrm{GHz}$ frequencies and implemented by means of impedance transformers (also called inverters) based on lattice networks and transmission line sections. The dual-band functionality of the proposed device is achieved thanks to the composite right/left handed (CRLH) behavior of the impedance transformers, able to provide $-90^{\circ}$ and $+90^{\circ}$ phase shift at the first and second design frequencies, respectively, of the divider. By using such combination of transmission line sections and lattice networks, the characteristic impedance of the impedance transformers is roughly constant over wide bandwidths, with the results of broad operating bands. To demonstrate the possibilities of the approach, a prototype device is designed, fabricated and characterized.
\end{abstract}

Keywords: metamaterials; lattice network; CRLH transmission line; power divider;

\section{Introduction}

The implementation of microwave devices able to exhibit their functionality at several frequencies has been a subject of an intensive research in recent years. One of the approaches for the implementation of dual-band, tri-band and (even) quad-band components uses artificial lines based on metamaterial concepts, also known as metamaterial transmission lines [1-3]. Such lines are transmission lines loaded with reactive elements, that is, inductors, capacitors, resonators, or a combination of them. Thanks to the presence of these reactive elements the lines exhibit enhanced design flexibility, and microwave devices with superior performance or with novel functionalities can be implemented by means of such artificial lines. Multiband components are examples of these new functional microwave devices. Typically, the multiband microwave components based on metamaterial transmission lines are composed of multiband impedance transformers, namely, transmission lines exhibiting $\pm 90^{\circ}$ phase shift at the design frequencies [4,5]. The key aspect to achieve such multiband functionality is the possibility to tailor the dispersion diagram of the considered artificial lines. Such controllability is not possible in ordinary lines. Indeed, metamaterial transmission lines have opened the path to the design of microwave components on the basis of impedance and dispersion engineering. By loading the lines with reactive elements, the dispersion diagram and the characteristic impedance of the resulting from the publishing process, such as peer review, editing, corrections, structural formatting, and other quality control mechanisms may not be reflected in this document. Changes may have been made to this work since it was submitted for publication. A definitive version was subsequently published in Vélez, P., Bonache, J. and Martín, F. "Dual and broadband power dividers at microwave frequencies based on composite right/left handed (CRLH) lattice networks" in Photonics and nanostructures, vol. 12, issue 4 (Aug. 2014), p. 269-278. DOI 10.1016/j-photonics.2014.05.006 
lines can be adjusted, so that certain specifications not easily achievable with conventional lines can be satisfied with metamaterial transmission lines. The simplest realistic artificial lines based on metamaterial concepts are the so-called composite right/left handed (CRLH) lines [6]. These lines can be implemented by loading a host line with series capacitors and shunt inductors (CL-loaded approach) [7-9], or by loading a host line with resonant elements, such as split ring resonators (SRRs) [10] or complementary split ring resonators (CSRRs) [11], and other reactive elements (shunt inductors or series capacitors). The latter approach has been designated as the resonant type approach [12-14].

Let us now focus the attention on the CL-loaded approach. The equivalent T-circuit model of the unit cell of these lines is depicted in Fig. 1. The dispersion diagram and the Bloch impedance (or ratio between voltage and current for the Bloch waves considering a periodic structure composed of such cells) of this structure can be inferred from the circuit model according to [15]

$$
\begin{gathered}
\cos \beta l=1+\frac{Z_{s}(\omega)}{Z_{p}(\omega)} \\
Z_{B}=\sqrt{Z_{s}(\omega)\left[Z_{s}(\omega)+2 Z_{p}(\omega)\right]}
\end{gathered}
$$

where $Z_{s}$ and $Z_{p}$ are the impedances of the series and shunt branch, respectively, of the T-circuit, $\beta$ is the phase constant and $l$ the unit cell length. If only one cell or few cells are considered, then expression (2) is identified with the image impedance, but both the Bloch and the image impedance are the equivalent of the characteristic impedance in ordinary lines. The resulting dispersion diagram exhibits a region (at low frequencies) where the phase and group velocities are antiparallel (left handed band), and a region (at higher frequencies) where both velocities have the same sign (right handed band). Hence these lines exhibit a CRLH behavior. In the left handed band, wave propagation is backward, whereas in the right handed band wave propagation is forward (i.e., as in ordinary lines). Typically, a band gap appears between the backward and forward transmission bands, unless the line is balanced. The balanced condition is achieved when the series and shunt resonance frequencies are identical [2], namely

$$
\omega_{s}=\frac{1}{\sqrt{L_{R} C_{L}}}=\frac{1}{\sqrt{L_{L} C_{R}}}=\omega_{p}
$$

In this case, the transition between the left handed and the right handed band is continuous, the phase velocity is infinite at the transition frequency, and the group velocity exhibits a finite value at that frequency. CRLH transmission lines described by the model of Fig. 1 exhibit a bandpass behavior. Interestingly, it is possible to design the line in order to exhibit the same absolute value of phase shift at two different frequencies $\left(f_{1}\right.$ and $\left.f_{2}\right)$, with the same values of the characteristic impedance. Considering that a single unit cell is used (in order to reduce dimensions), the conditions to univocally determine the four reactive elements of the circuit of Fig. 1, for an impedance transformer, are: 


$$
\begin{aligned}
& \chi_{s}\left(\omega_{1}\right)=-Z_{A} \\
& \chi_{p}\left(\omega_{1}\right)=+Z_{A} \\
& \chi_{s}\left(\omega_{2}\right)=+Z_{A} \\
& \chi_{p}\left(\omega_{2}\right)=-Z_{A}
\end{aligned}
$$

where $Z_{s}=j \chi_{s}, Z_{p}=j \chi_{p}, \omega_{1}=2 \pi f_{1}, \omega_{2}=2 \pi f_{2}, Z_{A}$ is the required characteristic impedance of the transformer (a real quantity) at the design frequencies, and the phase of the transformer is $-90^{\circ}$ at $f_{1}$ and $+90^{\circ}$ at $f_{2}$. Fig. 2 depicts the dispersion and characteristic impedance of the transformer with $Z_{A}=70.71 \Omega$ at $f_{l}=1 \mathrm{GHz}$ and $f_{2}=2 \mathrm{GHz}$.

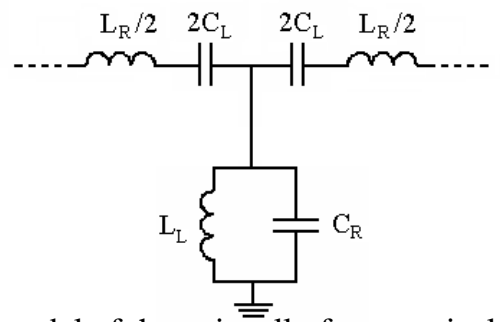

Fig. 1.Equivalent T-circuit model of the unit $\stackrel{\overline{\bar{c}}}{\mathrm{c}}$ ll of a canonical CRLH transmission line.
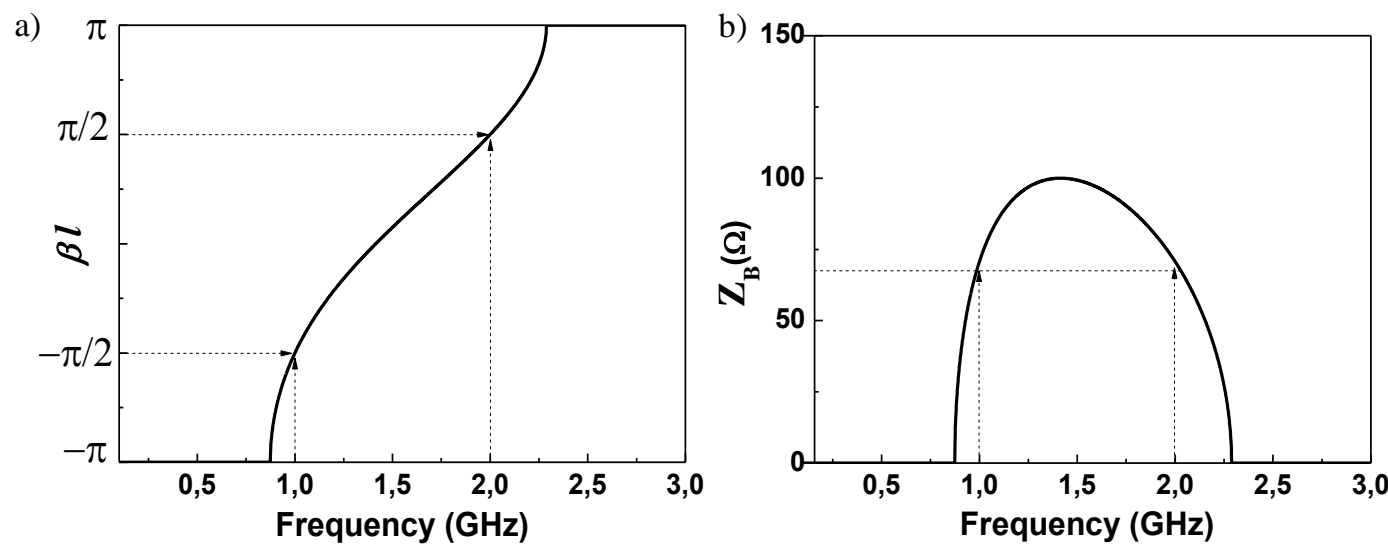

Fig. 2.Dispersion diagram (a) and characteristic impedance (b) of a dual-band impedance transformer with $Z_{A}=70.71 \Omega$, implemented by means of the circuit of Fig. 1 . The operating frequencies are $f_{l}=1 \mathrm{GHz}$ and $f_{2}=2 \mathrm{GHz}$. The element values that result by solving (4) are: $L_{L}=5.62 \mathrm{nH}, L_{R}=22.5 \mathrm{nH}, C_{L}=0.56 \mathrm{pF}$, $C_{R}=2.25 \mathrm{pF}$.

Many dual-band components have been implemented following this strategy $[5,16]$, and by increasing the number of reactive elements, quad-band components have also been reported (such components are based on the so-called extended, or generalized, CRLH transmission line concept [17-19], and several prototypes are reported in [20-22]). An important difference between CRLH lines and ordinary lines is the frequency dependence of the characteristic impedance. Whereas it is constant in conventional lines, it strongly depends on frequency in CRLH transmission lines. Note however that it is scarcely dependent on frequency in the vicinity of the transition frequency in balanced lines [2]. For this reason, many devices have been designed with multi-cell balanced structures, each one exhibiting a small phase shift at the required frequencies. Typically, the first (left handed) band in CRLH lines is narrow and the characteristic impedance in the vicinity of the frequency where the phase shift is $-90^{\circ}$ varies substantially with frequency. The result, when these lines are used for the 
implementation of dual-band devices based on impedance transformers, is a narrow band at the first operating frequency $[5,16]$.

In this paper, dual-band impedance transformers are implemented following a different approach: the use of lattice networks combined with transmission line sections. Through a proper design, these artificial lines can be designed to exhibit a CRLH behavior with a nearly frequency independent characteristic impedance. As will be shown, the designed dual-band device (a power divider) exhibits two operating bands with impedance matching over broad bandwidths, as compared to other reported dual-band components.

\section{Lattice networks exhibiting CRLH behavior: theory and state of the art}

The CRLH and the generalized CRLH transmission lines mentioned in the previous section support wave propagation in a limited frequency band. All-pass structures exhibiting left handed, right handed or CRLH wave transmission can be implemented by using the so-called lattice networks [23]. Indeed, all-pass CRLH transmission lines with arbitrary order can potentially be synthesized with these lattice network structures [24]. However, the practical implementation of these lattice (or X-type) networks is not straightforward, as will be later discussed.

Let us now consider an X-type unit cell like the one shown in Fig. 3(a), i.e., a lattice network, with impedance $Z_{s}$ in the series branches and $Z_{p}$ in the cross diagonal arms. By calculating the elements of the impedance matrix, this two-port network can be transformed to its equivalent T-circuit model, depicted in Fig. 3(b). Using (1) and (2), the phase constant and characteristic impedance of the generalized lattice network of Fig. 3(a) are given by:

$$
\begin{gathered}
\cos (\beta l)=\frac{Z_{p}+Z_{s}}{Z_{p}-Z_{s}} \\
Z_{B}=\sqrt{Z_{s} Z_{p}}
\end{gathered}
$$

a)

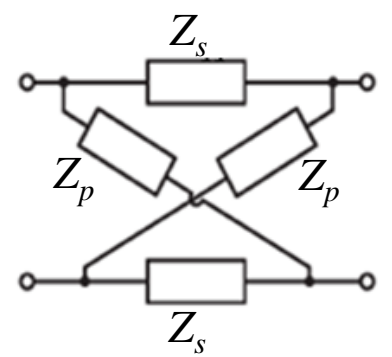

b)

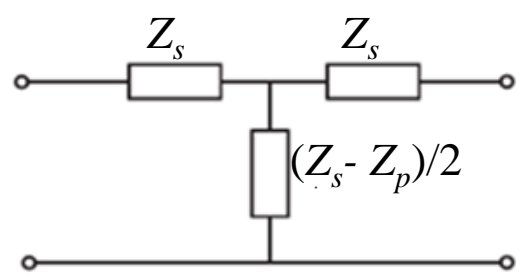

Fig. 3.Lattice-network unit cell (a) and its equivalent T-circuit model (b).

Let us now consider that the element of the series and cross branches is an inductor $\left(Z_{s}=\right.$ $\left.j \omega L_{R}\right)$ and a capacitor $\left(Z_{p}=-j / \omega C_{R}\right)$, respectively (Fig. 4a). Evaluation of (5) and (6) gives:

$$
\cos (\beta l)=\frac{1-L_{R} C_{R} \omega^{2}}{1+L_{R} C_{R} \omega^{2}}
$$




$$
Z_{B}=\sqrt{\frac{L}{C}}
$$

Inspection of (7) and (8) reveals that the network of Fig. 4(a) is an all-pass structure (the characteristic impedance is real and frequency independent, like the one of an ordinary transmission line), and that wave propagation is forward from DC up to (theoretically) unlimited frequencies (the dispersion diagram is also depicted in Fig. 4a). If the inductor and capacitor are interchanged (Fig. 4b), the characteristic impedance is also constant and given by (8), whereas the phase constant is found to be:

$$
\cos (\beta l)=-\frac{1-L_{L} C_{L} \omega^{2}}{1+L_{L} C_{L} \omega^{2}}
$$

and wave propagation is backward in the whole electromagnetic spectrum (see the dispersion diagram depicted in Fig. 4b). If the phase shift is small enough, the networks of Fig. 4(a) and (b) mimic a conventional line and a purely left-handed line, respectively.

By adequately choosing the reactive elements of the series and cross arms of the Xnetwork, all-pass CRLH structures of arbitrary order can be obtained [24]. For instance, Figs. 4 (c) and (d) show the lattice networks corresponding to order-2 all-pass CRLH and dual-CRLH lines, respectively. To obtain all-pass structures, it is necessary that the zeros and poles of the reactance of the series branch coincide with the zeros and poles of the susceptance of the cross branch. For the specific case of order-2 lattice networks, the condition is similar to the balance condition for T- or $\pi$-type CRLH transmission lines:

$$
\omega_{s} \equiv \frac{1}{\sqrt{L_{s} C_{s}}}=\frac{1}{\sqrt{L_{p} C_{p}}} \equiv \omega_{p}=\omega_{o}
$$

where $L_{s}, C_{s}$ and $L_{p}, C_{p}$ are the reactive elements of the series branch and cross branch, respectively, and $\omega_{o}$ is the transition frequency. For the network of Fig. 4(c), expression (5), subjected to (10), rewrites as:

$$
\cos (\beta l)=\frac{1-L_{s} C_{p} \omega^{2}\left(1-\frac{\omega_{o}^{2}}{\omega^{2}}\right)^{2}}{1+L_{s} C_{p} \omega^{2}\left(1-\frac{\omega_{o}^{2}}{\omega^{2}}\right)^{2}}
$$

whereas the dispersion relation for the network of Fig. 4(d) is:

$$
\cos (\beta l)=-\frac{1-L_{p} C_{s} \omega^{2}\left(1-\frac{\omega_{o}^{2}}{\omega^{2}}\right)^{2}}{1+L_{p} C_{s} \omega^{2}\left(1-\frac{\omega_{o}^{2}}{\omega^{2}}\right)^{2}}
$$


The dispersion curves are also depicted in Figs. 4(c) and (d). In both cases, the characteristic impedance is given by (8), with $L=L_{s}$ and $C=C_{p}$.
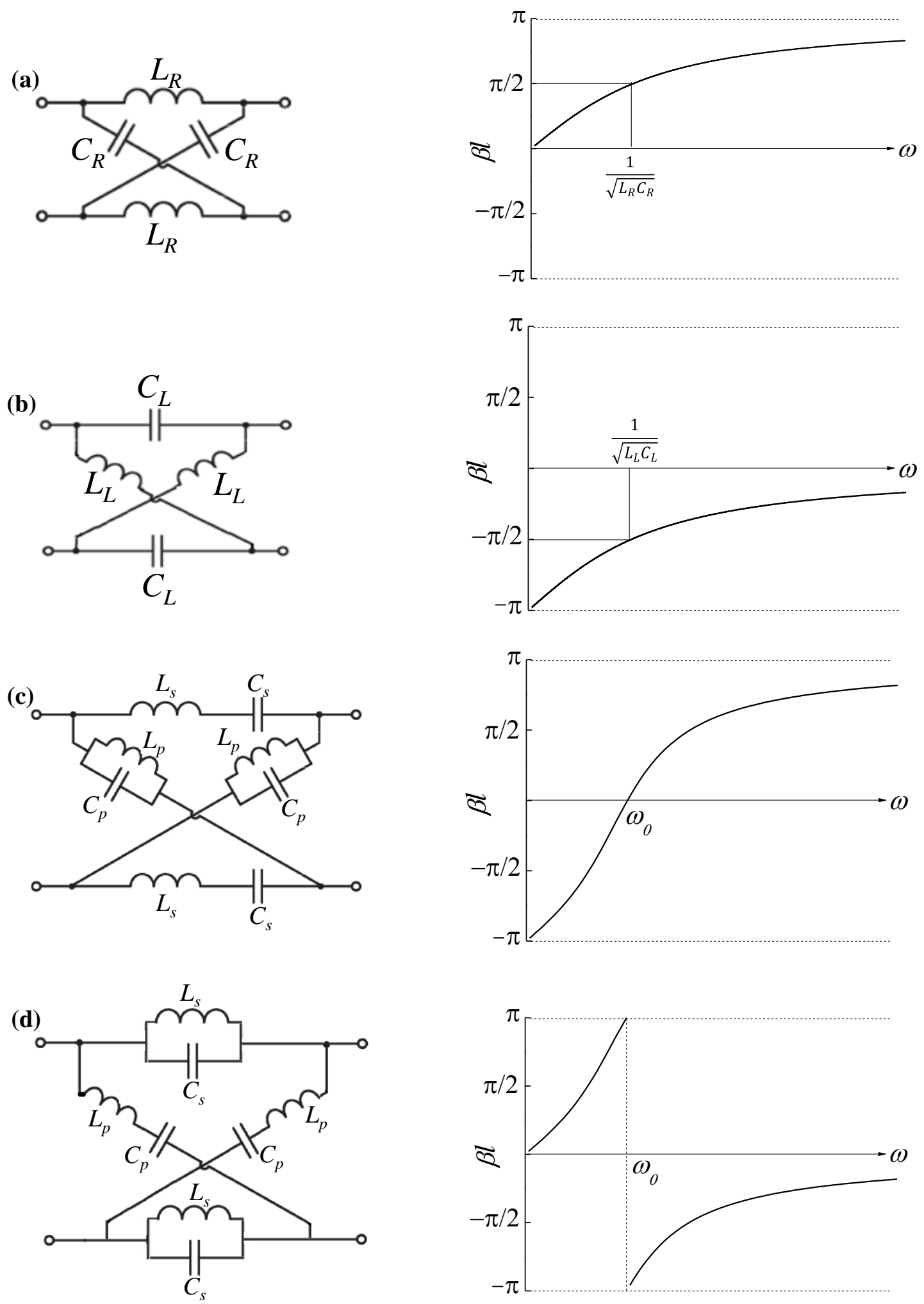

Fig. 4.Examples of lattice networks and their corresponding dispersion curves. (a) order-1 right handed; (b) order-1 left handed; (c) order-2 CRLH; (d) order-2 dual CRLH. 
From a practical viewpoint, the physical implementation of the order-2 lattice networks of Figs. 4(c) and (d) is not straightforward. An alternative to implement all pass order-2 CRLH lines is to combine purely left handed and purely right handed X-type cells, as depicted in Fig. 5(a). In this case, the following condition must be satisfied:

$$
\sqrt{\frac{L_{R}}{C_{R}}}=\sqrt{\frac{L_{L}}{C_{L}}} \equiv Z_{B}
$$

which gives the characteristic impedance of the whole all-pass network. The transition frequency is given by that frequency satisfying:

$$
\left|\arccos \left(\frac{1-L_{R} C_{R} \omega_{o}^{2}}{1+L_{R} C_{R} \omega_{o}^{2}}\right)\right|-\left|\arccos \left(-\frac{1-L_{L} C_{L} \omega_{o}^{2}}{1+L_{L} C_{L} \omega_{o}^{2}}\right)\right|=0
$$

namely:

$$
\omega_{o}=\frac{1}{\sqrt{L_{R} C_{L}}}=\frac{1}{\sqrt{L_{L} C_{R}}}
$$

In particular, if $L_{R} C_{R}=L_{L} C_{L}$ (which, according to 13, means that $L_{R}=L_{L}$ and $C_{R}=C_{L}$ ), the transition frequency can also be expressed as:

$$
\omega_{o}=\frac{1}{\sqrt{L_{R} C_{R}}}=\frac{1}{\sqrt{L_{L} C_{L}}}
$$

corresponding to the frequency where the purely left handed cell and the right handed cell experience a phase shift of $\beta l=-90^{\circ}$ and $\beta l=+90^{\circ}$, respectively (thus providing an overall phase shift of $\beta l=0^{\circ}$ ). Fig. 5(b) shows the dispersion diagram corresponding to the structure of Fig. 5(a) for the indicated element values.
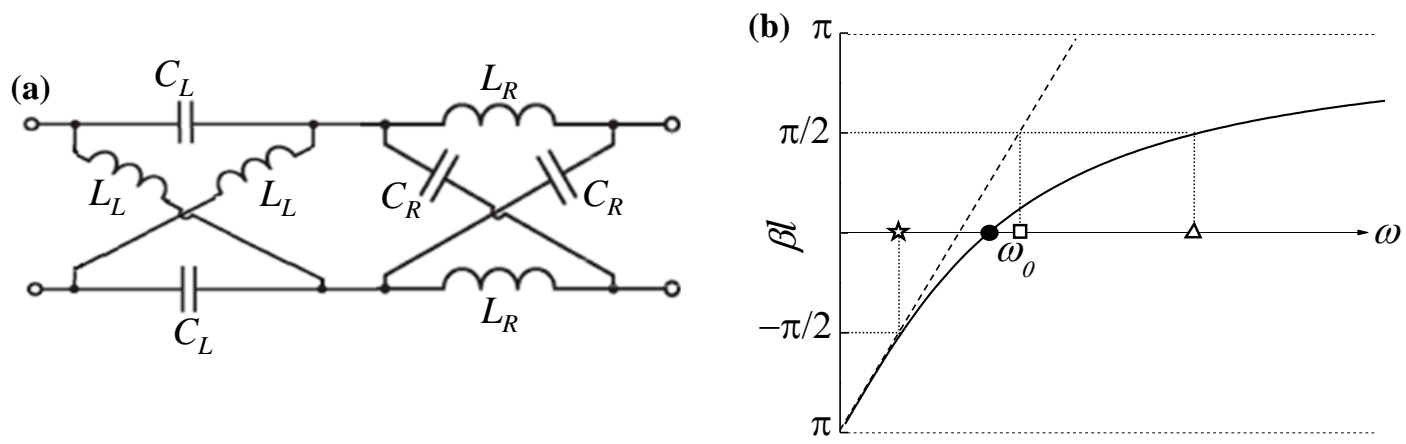

Fig. 5. CRLH artificial transmission line unit cell consisting of two cascaded order-1 X-type right handed and left handed cells (a), and dispersion diagram (b). Elements are: $C_{L}=C_{R}=1.061 \mathrm{pF}$ and $L_{L}=L_{R}=$ $2.652 \mathrm{nH}\left(f_{o}=3 \mathrm{GHz}\right.$, and it is indicated by a bold circle in $\left.\mathrm{b}\right)$.

It is worth mentioning that, in spite that the networks of Figs. 4(c) and 5(a) exhibit similar dispersion curves; there is not an element transformation that makes them equivalent. Notice that the dispersion relation (with the frequency variable in the $x$-axis) for the structure of Fig. 5(a) is a concave function at all frequencies. However, for the 
order-2 CRLH X-type cell of Fig. 4(c), the dispersion relation is convex for frequencies below $\omega_{o}$, and concave above that frequency (i.e., there is an inflexion point at $\omega_{o}$ ). Although the synthesis of a cascaded order-1 left handed and right handed X-type cells is simpler than an order-2 CRLH lattice network, the former structure may present certain limitations due to the curvature of its dispersion relation. For instance, it is not possible to implement dual-band components based on dual-band $\left( \pm 90^{\circ}\right)$ impedance inverters implemented with single unit cells (like the one depicted in Fig. 5a) with a ratio of operating frequencies smaller than 3 . The reason is that, due to the curvature of the dispersion diagram (see Fig. 5b), the second operation frequency (indicated by a triangle in Fig. 5b) is always above the one indicated by a square, this one being the second operating frequency if the dispersion curve was a straight line (dashed line in Fig. 5b). Notice that this frequency is exactly three times the one indicated by a star (the first operating frequency). Hence the above statement is graphically demonstrated.

The synthesis of CRLH all-pass artificial lines can be further simplified by cascading an $\mathrm{X}$-type left handed unit cell with a transmission line section with identical characteristic impedance, as depicted in Fig. 6(a), or with a pair of transmission lines sections (at the input and output ports of the left handed X-type cell). A typical dispersion curve for the structure of Fig 6(a) is depicted in Fig. 6(b). In this case, due to the presence of a distributed element, the phase of the structure grows indefinitely.

(a)

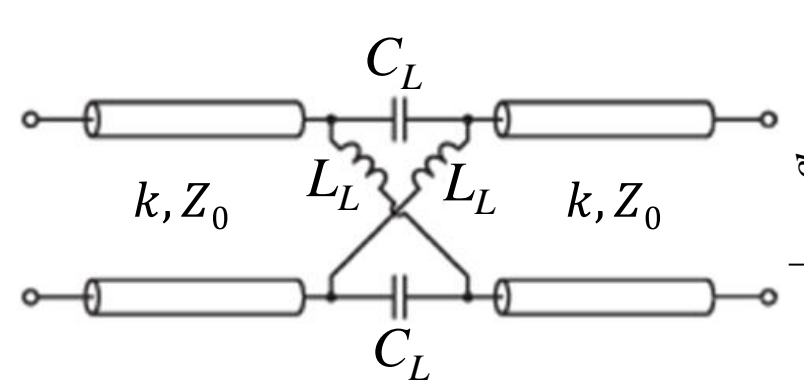

(b)

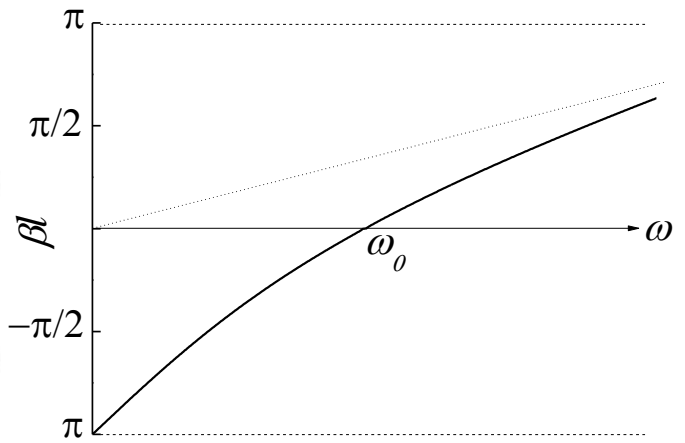

Fig. 6. CRLH artificial transmission line unit cell consisting of an order-1 X-type left handed cell cascaded to an ordinary transmission line section with phase constant $k$ and characteristic impedance $Z_{o}$.

Concerning the implementation, X-type networks are differential structures with cross branches. Therefore, as mentioned above, their synthesis is not simple. CRLH artificial lines were implemented by Bongard et al. [25-27] by combining X-type left handed cells and transmission line sections. The structure reported in [27] utilizes paired strips technology with two additional metal levels to implement the series capacitances of the left handed X-type cells, and via holes to implement the cross inductances. The structure, designed to exhibit a transition frequency at $f_{o}=6 \mathrm{GHz}$, exhibits very broad bands at both sides of $f_{o}$.

Using the unit cell structure of Fig. 6(a), a broad-band (matched from DC to millimeter waves) silicon-integrated CRLH transmission line using a monolayer CPW host line was proposed in [28]. The measurements carried out in [28] demonstrate a balanced CRLH behavior from $5 \mathrm{GHz}$ up to $35 \mathrm{GHz}$, with a transition frequency at $f_{o}=20 \mathrm{GHz}$. 
The interesting aspect of the structure of [28] is that it is implemented using a single metal layer. Since the series capacitances must be implemented in both conductors of the transmission line, the ground conductors of the host CPW must be of finite width. The required capacitances were realized by interdigital capacitors, whereas for the implementation of the crossed inductors, the solution proposed in [28] was to implement each of the inductances in a different slot of the CPW. Since the resulting structure is strongly asymmetrical, the excitation of the odd parasitic mode was prevented by the use of the bridges. Thus, the structure indeed uses two metal levels. Other X-type left handed, right handed and CRLH planar structures implemented by using two metal levels are reported in [29].

It is worth mentioning that from the equivalence between terminated coupled line sections and lattice networks [30], another approach for the physical implementation of lattice network based artificial CRLH lines was presented in [24]. The unit cell is based on the coupled-microstrip Schiffman section [31], which is an easily implementable structure that does not require the use of via-holes or air bridges, as opposed to the cells presented in [25-29]. Indeed, coupled-line sections were earlier used in [32,33] to obtain CLRH transmission lines, but with limited performance due to the different even- and odd-mode phase velocities of the coupled lines. This issue was satisfactorily solved in [24]. Thus, terminated coupled line sections offer a simple approach for the implementation of lattice network CRLH transmission lines. Obviously, this is a fully distributed approach that inherently increases line size as compared to lattice network CRLH lines based on lumped or semilumped (planar) elements.

\section{Dual-band power dividers based on left handed lattice networks and transmission line sections}

The dual-band power divider presented in this paper is implemented with a pair of dualband $70.71 \Omega$ impedance transformers based on the CRLH structure of Fig. 6, i.e., a combination of a purely left handed lattice network and a transmission line section. The schematic of this divider is depicted in Fig. 7. The impedance of the impedance transformers $\left(Z_{A}=70.71 \Omega\right)$ has been set to this value in order to achieve good input matching, considering reference impedances of $Z_{o}=50 \Omega$ at the ports. At the design frequencies the phase shift of the impedance transformers is $\pm 90^{\circ}$, and hence the impedance seen at the input port of each transformers, given by [15]

$$
Z_{\text {in }, T}=\frac{Z_{A}^{2}}{Z_{o}}
$$

is $100 \Omega$, and the impedance seen from port 1 is $Z_{i n}=50 \Omega$ at the design frequencies. 


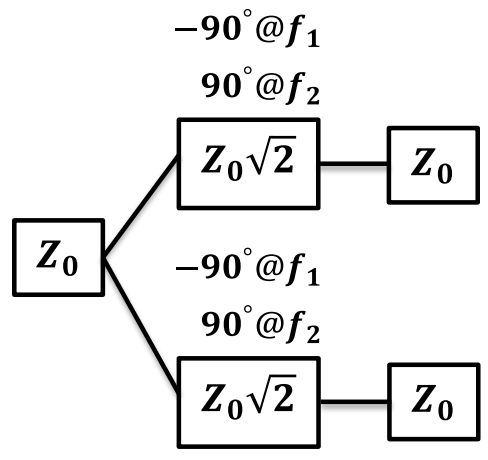

Fig. 7.Schematic of the dual-band power divider.

By choosing the operating frequencies as $f_{1}=1.7 \mathrm{GHz}$ and $f_{2}=5.4 \mathrm{GHz}$, the conditions to implement the impedance transformers are:

$$
\begin{gathered}
Z_{A}=\sqrt{\frac{L_{L}}{C_{L}}}=70.71 \Omega \\
\arccos \left(-\frac{1-L_{L} C_{L} \omega_{1}^{2}}{1+L_{L} C_{L} \omega_{1}^{2}}\right)+\phi_{T L}\left(\omega_{1}\right)=-90^{\circ} \\
\arccos \left(-\frac{1-L_{L} C_{L} \omega_{2}^{2}}{1+L_{L} C_{L} \omega_{2}^{2}}\right)+\phi_{T L}\left(\omega_{2}\right)=+90^{\circ}
\end{gathered}
$$

where $\phi_{T L}(\omega)$ is the phase of the transmission line section, given by

$$
\phi_{T L}(\omega)=k l_{T L}=\frac{\omega}{v_{p}} l_{T L}
$$

$v_{p}$ and $l_{T L}$ being the phase velocity and length of the transmission line section, respectively.

Notice that equations (18)-(20) have three unknowns $\left(L_{L}, C_{L}\right.$ and $\left.l_{T L}\right)$ that can be univocally determined. Solving (18)-(20) with the design frequencies given above, the unknowns are found to be: $L_{L}=2.09 \mathrm{nH}, C_{L}=0.419 \mathrm{pF}$, and $l_{T L} / v_{p}=19.18 \mathrm{ps}$ (obviously, the phase velocity depends on the effective dielectric constant of the considered line). For the implementation of the divider using the inferred CRLH impedance transformers, we have considered the topology depicted in Fig. 8. Basically, the series capacitances of the left-handed lattice network are implemented by means of metallic gaps, whereas the crossed inductances are implemented by means of narrows strips with vias (with $0.15 \mathrm{~mm}$ radius) in order to connect the upper metal with the lower metal. Transmission line sections with the required values $\left(Z_{B}=70.71\right.$ and $\left.l_{T L} / 2 v_{p}=9.59 \mathrm{ps}\right)$ are added at both sides of the $X$-type left handed unit cell to provide the needed phase shift. This transmission line sections are implemented by means of double sided parallel strip line (DSPSL) [34] with an offset equal to $0.66 \mathrm{~mm}$ (this gives the required impedance) and connected to the X-type left handed lattice network with tapered line sections, that have been optimized to achieve the required phase response (the tapper lines are necessary to 
connect the lines to the X-type network, which has the indicated shape in order to accommodate the inductive vias, and to provide the required capacitive values).

(a)

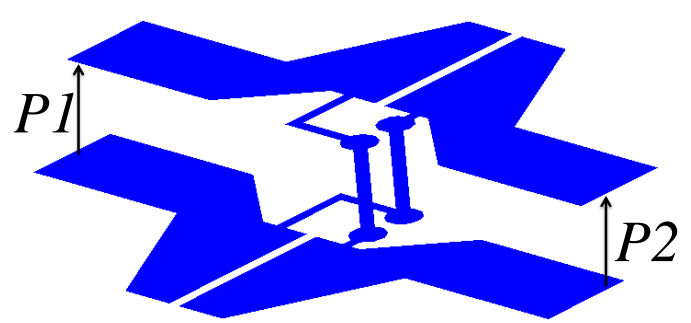

(b)

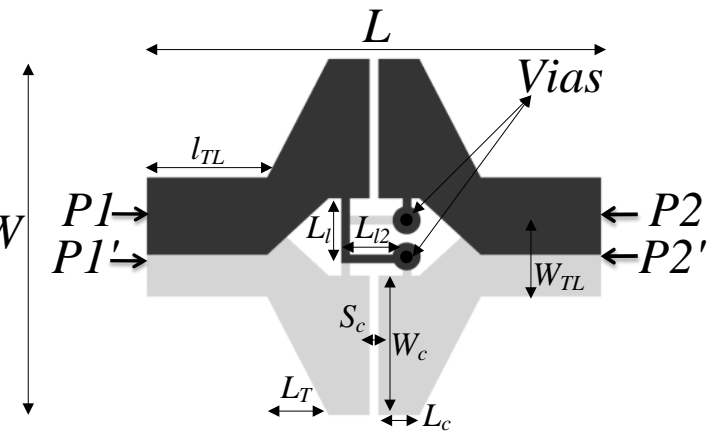

Fig.8. 3D view of the dual-band impedance inverter based on an X-type left handed lattice sandwiched between two transmission line sections (a), and top view with ports and relevant dimensions indicated (b). The lower metal is depicted in grey. Dimensions are: $W=6.6 \mathrm{~mm}, L=8.4 \mathrm{~mm}, W_{c}=2.6 \mathrm{~mm}, L_{c}=$ $0.75 \mathrm{~mm}, S_{c}=0.16 \mathrm{~mm}, l_{L T}=2.22 \mathrm{~mm}, L_{T}=1.13 \mathrm{~mm}, W_{T L}=1.43 \mathrm{~mm}, L_{l}=1.2 \mathrm{~mm}$ and $L_{l 2}=0.95 \mathrm{~mm}$. The width of the narrow strips is $0.16 \mathrm{~mm}$.

Fig. 9 depicts the dispersion diagram and Bloch impedance of the dual-band inverter of Fig. 8 inferred from the schematic of Fig. 6 (by considering the element values and transmission line parameters of the structure) and also from the electromagnetic response (obtained by means of the Agilent Momentum commercial software). The considered substrate is the Rogers RO3010 with dielectric constant $\varepsilon_{r}=10.2$ and thickness $h=1.27 \mathrm{~mm}$. As expected, the required phase shift at the operating frequencies is achieved. Since the structure in not perfectly balanced, a singularity is observed around the transition frequency $(3.4 \mathrm{GHz})$. This singularity appears also in the characteristic impedance, but it is nearly frequency independent in the vicinity of the operating frequencies.
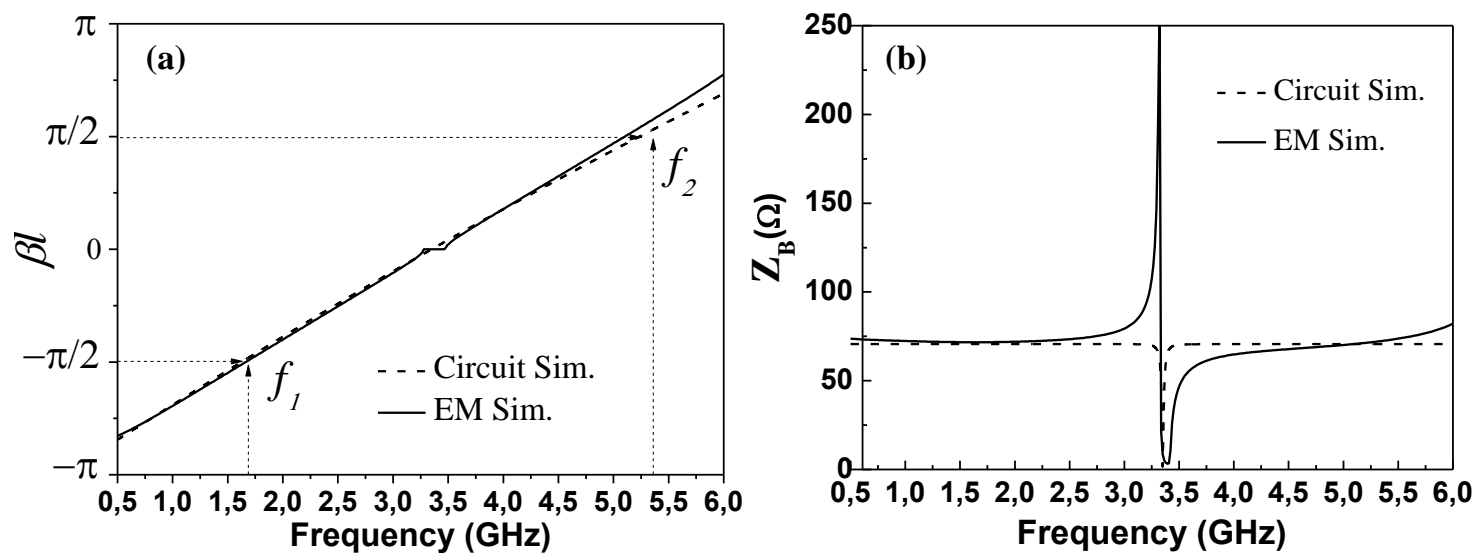

Fig. 9. Dispersion diagram (a) and characteristic impedance (b) of the dual-band impedance transformer, with $Z_{A}=70.71 \Omega$, implemented by means of the topology of Fig. 8 .

The designed and fabricated dual-band power divider with final dimensions is depicted in Fig. 10. Notice that the $50 \Omega$ access line in port 1 is implemented by means of a double sided parallel strip line. 


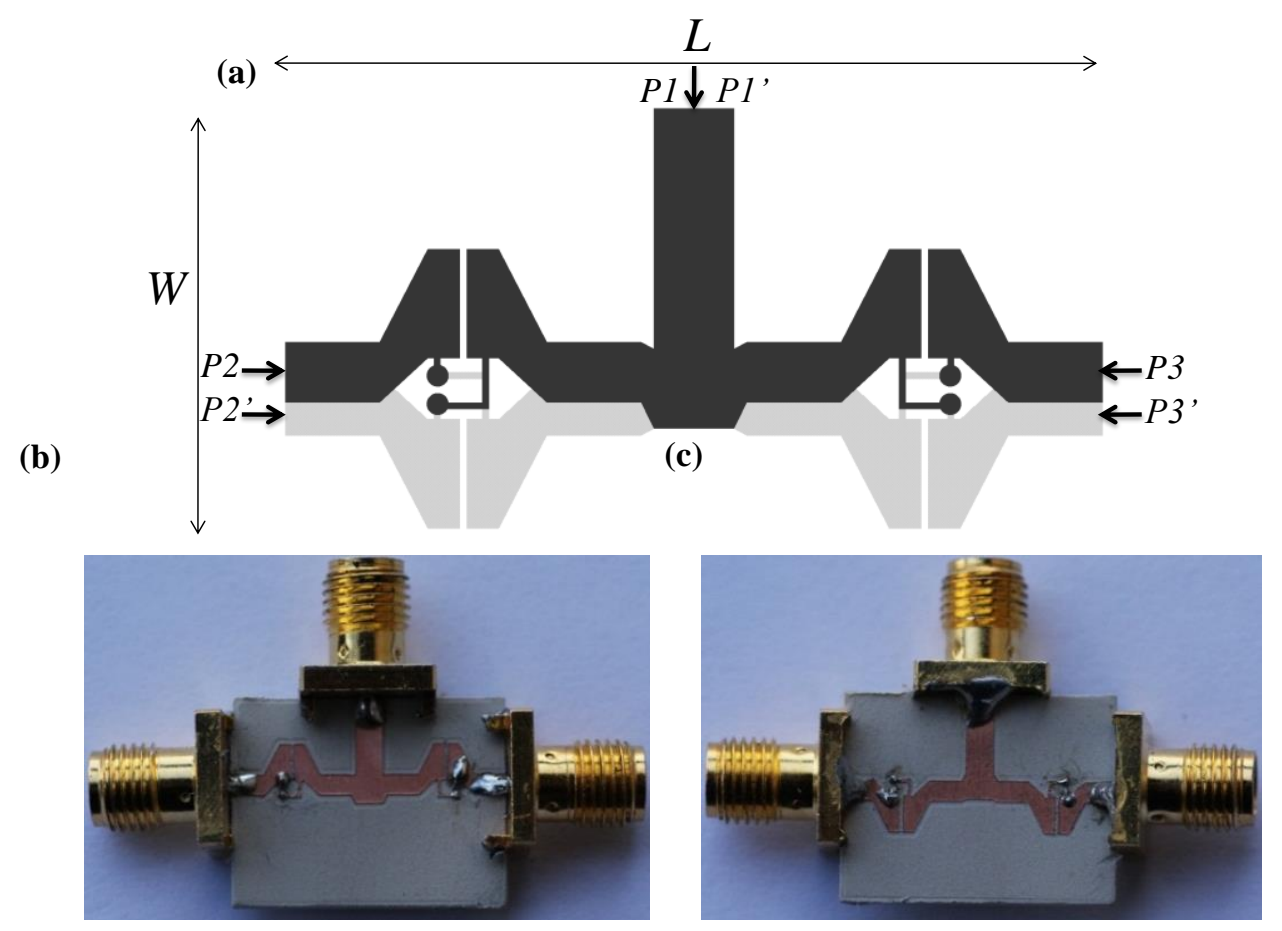

Fig. 10. Topology (a) and photograph, top (b) and bottom (c), of the fabricated dual band power divider. Dimensions are: $W=9.9 \mathrm{~mm}, L=19.2 \mathrm{~mm}$.

The circuit simulation, electromagnetic simulation and measured insertion loss $\left(S_{31}\right.$ is very similar to $S_{21}$ and is not depicted) and return loss $\left(S_{11}\right)$ are depicted in Fig.11. The agreement between circuit, electromagnetic simulation and measurement is reasonable. The measured insertion loss (notice that the ideal value is $3 \mathrm{~dB}$ ) and return loss at the design frequencies are $I L\left(f_{1}\right)=3.6 \mathrm{~dB}, R L\left(f_{1}\right)=21.3 \mathrm{~dB}, I L\left(f_{2}\right)=3.7 \mathrm{~dB}$, and $R L\left(f_{2}\right)=$ $20.7 \mathrm{~dB}$. Device dimensions are $0.14 \lambda_{g} \times 0.28 \lambda_{g}$, where $\lambda_{g}$ is the guided wavelength at $f_{1}$. As it was mentioned before, this type of structures exhibits (ideally) frequency independent characteristic impedance, resulting in broader bandwidths, as compared to other CRLH based circuits. Indeed, the designed device exhibits a roughly constant simulated insertion loss and the matching level is better than $10 \mathrm{~dB}$ in very broad frequency bands in the vicinity of the design frequencies.

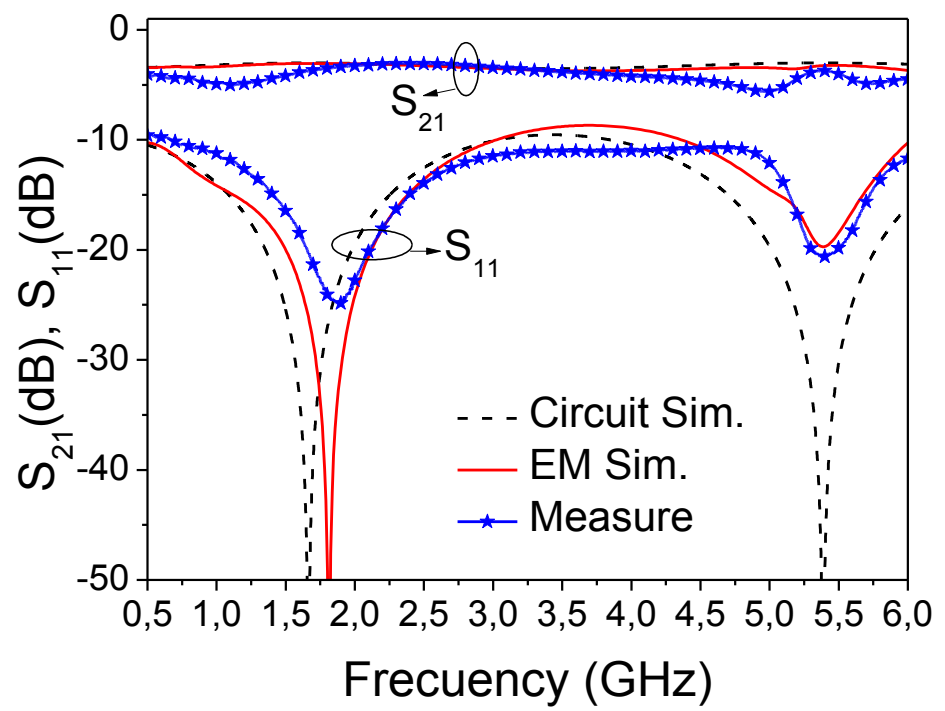

Fig. 11. Frequency response of the fabricated dual-band power divider designed to operate at $f_{l}=1.7 \mathrm{GHz}$ and $f_{2}=5.4 \mathrm{GHz}(\mathrm{b}) . \mathrm{S}_{31}$ has not been included since this is similar to $\mathrm{S}_{21}$. 


\section{Conclusion}

In this paper, the potentiality of lattice network unit cells for the implementation of enhanced bandwidth microwave components has been demonstrated. These all-pass networks can be designed to exhibit left handed, right handed or composite right/left handed (CRLH) behaviour. Specifically in this work, to ease design and synthesis, the authors have combined a left handed lattice unit cell with a pair of ordinary transmissions line sections in order to implement a dual-band impedance inverter with broad operating bandwidths. This inverter has been then used for the implementation of a dual-band T-junction power divider. Thanks to the nearly constant characteristic impedance of the inverters in the vicinity of the design frequencies, the divider has been found to exhibit good matching level and power splitting over wide bands. To the authors knowledge, this is the first dual-band power divider implemented by means of CRLH structures (impedance inverters) that combine X-type networks and transmission line sections.

\section{Acknowledgements}

This work has been supported by MINECO-Spain (projects TEC2010-17512 and CSD2008-00066), and by AGAUR-Generalitat de Catalunya, through project 2014SGR-157. Paris Vélez is in debt to MINECO (Spain) for supporting his work through the FPU grant AP2010-0467.

\section{References}

1. G.V. Eleftheriades and K.G. Balmain, Negative refraction metamaterials: fundamental principles and applications, John Wiley \& Sons, Inc, New Jersey 2005.

2. C. Caloz and T. Itoh, Electromagnetic Metamaterials: Transmission Line Theory and Microwave Applications, John Wiley \& Sons, 2006.

3. R. Marqués, F. Martín and M. Sorolla, Metamaterials with negative parameters: theory, design and microwave applications, John Wiley \& Sons, Inc, New Jersey 2008.

4. I.H. Lin, M. De Vincentis, C. Caloz and T. Itoh, "Arbitrary dual-band components using composite rigth/left handed transmisión lines", IEEE Trans. Microwave Theory and Techniques, vol. 52, pp. 1142-1149, April 2004

5. J. Bonache, G. Sisó, M. Gil, A. Iniesta, J. García-Rincón and F. Martín, “Application of composite right/left handed (CRLH) transmission lines based on complementary split ring resonators (CSRRs) to the design of dual band microwave components", IEEE Microwave and Wireless Components Letters, vol. 18, pp. 524-526, August 2008.

6. C. Caloz and T. Itoh, "Novel microwave devices and structures based on the transmission line approach of metamaterials", IEEE-MTT Int'l Microwave Symp, vol. 1 Philadelphia, PA, pp. 195-198, June 2003.

7. A. K. Iyer and G. V. Eleftheriades. "Negative refractive index metamaterials supporting 2-D waves," in IEEE-MTT Int'l Microwave Symp., vol. 2, Seattle, WA, pp. 412- 415, June 2002.

8. A. A. Oliner. "A periodic-structure negative-refractive-index medium without resonant elements," in URSI Digest, IEEE-AP-S USNC/URSI National Radio Science Meeting, San Antonio, TX, pp. 41, June 2002.

9. C. Caloz and T. Itoh. "Application of the transmission line theory of left-handed (LH) materials to the realization of a microstrip LH transmission line," in Proc.IEEE-AP-S USNC/URSI National Radio Science Meeting, vol. 2, San Antonio, TX, pp. 412-415, June 2002.

10. J.B. Pendry, A.J. Holden, D.J. Robbins and W.J. Stewart, "Magnetism from conductors and enhanced nonlinear phenomena", IEEE Transactions Microwave Theory Tech., vol. 47, pp. 2075-2084, Nov. 1999. 
11.F. Falcone, T. Lopetegi, J.D. Baena, R. Marqués, F. Martín and M. Sorolla, "Effective negative- $\varepsilon$ stop-band microstrip lines based on complementary split ring resonators", IEEE Microwave and Wireless Components Letters, vol. 14, pp. 280-282, June 2004.

12. F. Martín, F. Falcone, J. Bonache, R. Marqués and M. Sorolla, "Split ring resonator based left handed coplanar waveguide”, Appl. Phys. Lett., vol. 83, pp. 4652-4654, December 2003.

13. J.D. Baena, J. Bonache, F. Martín, R. Marqués, F. Falcone, T. Lopetegi, M.A.G. Laso, J. García, I Gil, M. Flores-Portillo and M. Sorolla, "Equivalent circuit models for split ring resonators and complementary split rings resonators coupled to planar transmission lines", IEEE Transactions on Microwave Theory and Techniques, vol. 53, pp. 1451-1461, April 2005.

14. M. Durán-Sindreu, A. Vélez, G. Sisó, J. Selga, P. Vélez, J. Bonache, and F. Martín "Recent advances in metamaterial transmission lines based on split rings", Proceedings of the IEEE, vol. 99, pp. 17011710, October 2011.

15. D.M. Pozar, Microwave Engineering, Addison Wesley: New York; 1993.

16. M. Durán-Sindreu, A. Vélez, F. Aznar, G. Sisó, J. Bonache and F. Martín, “Application of Open Split Ring Resonators and Open Complementary Split Ring Resonators to the Synthesis of Artificial Transmission Lines and Microwave Passive Components", IEEE Trans. Microwave Theory and Techniques, vol. 57, pp. 3395-3403, Dec. 2009.

17. A. Rennings, S. Otto, J. Mosig, C. Caloz, and I. Wolff, "Extended composite right/left-handed metamaterial and its application as quadband quarter-wavelength transmission line," in Proc. AsiaPacific Microw. Conf. (APMC), Yokohama, Japan, Dec. 2006, pp. 1405-1408.

18. G. V. Eleftheriades, "A generalized negative-refractive-index transmission-line (NRL-TL) metamaterial for dual-band and quad-band applications," IEEE Microw. Wireless Compon. Lett., vol. 17, no. 6, pp. 415-417, Jun. 2007.

19. G. Sisó, M. Gil, J. Bonache and F. Martín, "Generalized model for multi-band metamaterial transmission lines”, IEEE Microwave and Wireless Components Letters, vol. 18, pp. 728-730, November 2008.

20. A. C. Papanastasiou, G. E. Georghiou, and G. V. Eleftheriades, "A quad-band Wilkinson power divider using generalized NRI transmission lines," IEEE Microw. WirelessCompon. Lett., vol. 18, no. 8, pp. 521-523, Aug. 2008.

21. M. Durán-Sindreu, G. Sisó, J. Bonacheand F. Martín, "Planar multi-band microwave components based on the generalized composite right/left handed transmission line concept", IEEE Transactions on Microwave Theory and Techniques, vol. 58, no 12, pp. 3882-3891, Dec. 2010.

22. M. Durán-Sindreu, J. Bonache, F. Martín and T. Itoh, "Single-Layer Fully-Planar ExtendedComposite Right/Left Handed Transmission Lines based on Substrate Integrated Waveguides for Dual-Band and Quad-Band Applications", International Journal of Microwave and Wireless Technologies, vol. 5 pp. 213-229, June 2013.

23. O.J. Zobel, "Theory and design of uniform and composite electric wave filters", Bell Systems Technical Journal, vol. 2, pp. 1-46, Jan. 1923.

24. J. Esteban, C. Camacho-Peñalosa, J.E. Page, and T.M. Martín-Guerrero, "Generalized lattice networkbased balanced composite right-/left-handed transmission lines", IEEE Trans. MicrowaveTheory and Techniques, vol. 60(8), pp. 2385-2393, Aug. 2012.

25. F. Bongard and J. R. Mosig, "A novel composite right/left-handed unit cell and potential antenna applications," Proc. IEEE Antennas Propag. Soc. Int. Symp., Jul. 2008, pp. 1-4.

26. F. Bongard, J. Perruisseau-Carrier, and J. R. Mosig, "A novel composite right/left-handed unit cell based on a lattice topology: Theory and applications," Proc. Metamaterials, Pamplona, Spain, Sep. 2008, pp. 338-340.

27. F. Bongard, J. Perruisseau-Carrier, and J. R. Mosig, "Enhanced CRLH transmission line performances using a lattice network unit cell," IEEE Microw.WirelessCompon. Lett., vol. 19, no. 7, pp. 431-433, Jul. 2009.

28. J. Perruisseau-Carrier, F. Bongard, M. Fernandez-Bolaños, and A.M. Ionescu, "A microfabricated 1-D metamaterial unit cell matched from DC to millimeter-waves", IEEE Microw. Wireless Compon. Lett., vol. 21, no. 9, pp. 456-458, Sept. 2011.

29. P. Vélez, M. Durán-Sindreu, J. Bonache and F. Martín, "Compact right-handed (RH) and left-handed (LH) lattice-network unit cells implemented in monolayer printed circuits", Asia Pacific Microwave Conference, Melbourne (Australia), Dec. 2011, pp. 534-537.

30. J.E. Page, J. Esteban, and C. Camacho-Peñalosa, "Lattice equivalent circuits of transmission-line and coupled-line sections", IEEE Trans. Microwave Theory and Techniques, vol. 59(10), pp. 2422-2430, Oct. 2011

31. B. M. Schiffman, "A new class of broadband microwave 90-degree phase shifters", IRE Trans. Microw. Theory Tech., vol. MTT-6, no. 2, pp. 232-237, Apr. 1958. 
32. A. M. E. Safwat, "Microstrip coupled line composite right/left-handed unit cell", IEEE Microw. Wireless Compon. Lett., vol. 19, no. 7, pp. 434-436, Jul. 2009.

33. A. E. Fouda, A. M. E. Safwat, and H. El-Hennawy, "On the applications of the coupled-line composite right/left-handed unit cell”, IEEE Trans. Microw. Theory Tech., vol. 58, no. 6, pp. 15841591, Jun. 2010.

34. W. Che, L. Gu and Y. L. Chow, "Formula derivation and verification of characteristic impedance for double-sided parallel strip line (DSPSL)", IEEE Microw. Wireless Compon. Lett., vol. 20, no. 6, pp. 304-306, Jun. 2010. 